\title{
Acute promyelocytic leukemia following autologous bone marrow-derived mesenchymal stem cell transplantation for traumatic brain injury: A case report
}

\author{
KUI SONG ${ }^{1,2}$, WEICHAO $\mathrm{LI}^{2}$ and $\mathrm{MIN} \mathrm{LI}^{3}$ \\ ${ }^{1}$ Department of Hematology, The First Affiliated Hospital of Jishou University, Jishou, Hunan 416000; \\ ${ }^{2}$ Department of Hematology, Zhongshan City People's Hospital, Zhongshan, Guangdong 528400; \\ ${ }^{3}$ Department of Pharmacy, The First Affiliated Hospital of Jishou University, Jishou, Hunan 416000, P.R. China
}

Received October 6, 2014; Accepted June 25, 2015

DOI: $10.3892 / \mathrm{ol} .2015 .3636$

\begin{abstract}
Acute myeloid leukemia (AML) is an extremely rare complication that can be observed following mesenchymal stem cells (MSC) transplantation for traumatic brain injury (TBI). Few cases have been reported thus far. The present study reports a case of acute promyelocytic leukemia (APL) following MSC transplantation in a 36-year-old female. The patient presented with a fever and dermatorrhagia with an associative abnormal coagulation test almost 2 months after the MSC transplantation for TBI. The routine blood test, bone marrow (BM) test, and examination of the promyelocytic leukemia/retinoic acid receptor- $\alpha$ and mixed lineage leukemia chimeric genes confirmed the diagnosis of APL and disseminated intravascular coagulation (DIC). The patient was treated with all-trans retinoic acid to induce remission and cryoprecipitate transfusion for the coagulation abnormality. However, the patient succumbed to the DIC shortly after treatment. To the best of our knowledge, this is the first report of the early occurrence of APL in a patient who received autologous BM-derived MSC therapy for TBI.
\end{abstract}

\section{Introduction}

Traumatic brain injury (TBI) is a worldwide health problem, causing mortality and permanent disability, including impaired attention and poor executive function as a result of neurocognitive deficits (1). The annual incidence and mortality rates of TBI in Europe are 235 and 15 cases

Correspondence to: Dr Min Li, Department of Pharmacy, The First Affiliated Hospital of Jishou University, Shiji Avenue, Jishou, Henan 416000, P.R. China

E-mail: zs_hematology@163.com

Key words: acute promyelocytic leukemia, mesenchymal stem cell, transplantation, traumatic brain injury per 100,000 individuals, respectively (2). However, the management for patients with TBI has recently improved substantially (3). However, the prognosis for patients with severe TBI remains poor, with results such as disturbance of consciousness and motor disorders. Stem cells have been suggested to be of potential for the repair of the damaged nervous system (4). Mesenchymal stem cell (MSC)-based cellular therapy has been studied in early-phase clinical trials to improve the effects of central nervous system (CNS) injuries (5). Human bone marrow MSCs (BMMSCs) have been widely studied, as they are relatively easy to access and they have potential to differentiate into the osteogenic, adipogenic and chondrogenic lineages, and into hepatocytes, cardiomyocytes, neurons and other types of tissues or cells (6). Previous studies have reported that transplanted BMMSCs accelerated neuroplasticity and facilitated neuronal regeneration, as well as functional recovery (7). Currently, autologous BM-derived stem cell transplantation $\mathrm{s}$ is one of the most common procedures in stem cell research. However, complications for autologous MSC therapy, including a few serious complications, have continued to be reported (8). As MSCs are multipotent, the issue of safety requires further consideration. When these cells are implanted, it may cause a low prevalence of neoplasms (5). The present study reports a case in which acute promyelocytic leukemia (APL) developed following treatment with autologous BMMSC transplantation for TBI. Written informed consent was obtained from the patient.

\section{Case report}

A 36-year-old female was admitted to a local hospital due to a severe traumatic brain injury caused by a traffic accident in December 2009. Paralysis of the lower limbs and the hands was observed following the surgery for the trauma. In early April 2011, the patient was treated with autologous BMMSCs transplantation by subarachnoid space injection. Following transplantation, the patient developed skin ecchymosis that persisted for 1 week and a fever that lasted for 3 days, and was subsequently transferred to Zhongshan City People's Hospital,(Zhongshan, China) on May 20, 2011. The routine 


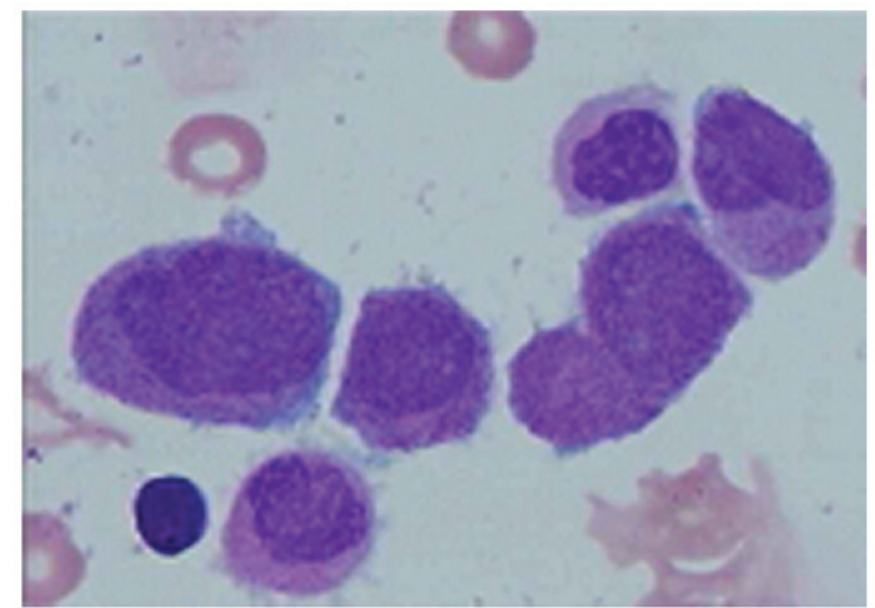

Figure 1. Bone marrow smear stained with Wright-Giemsa prior to treatment with all-trans retinoic acid showing the presence of promyelocytes (3\% myeloblasts and $76.5 \%$ promyelocytes).

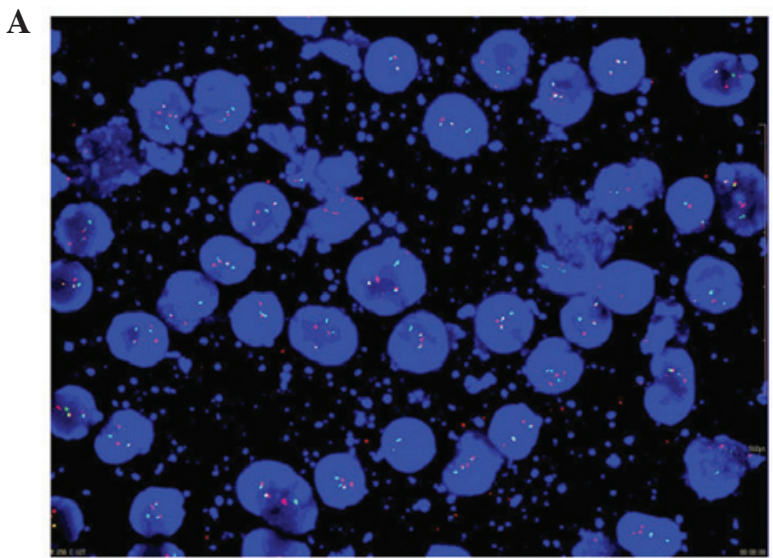

$\mathbf{B}$

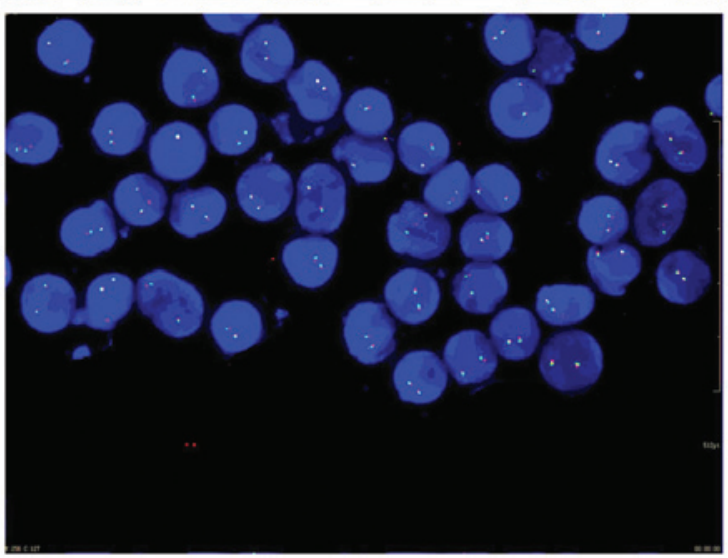

Figure 2. Fluorescence in situ hybridization (FISH) analysis of metaphase nuclei was performed with a specific dual-color DNA probe to count 200 bone marrow interphase cells; promyelocytic leukemia/retinoic acid receptor- $\alpha$ (PML/RARA) and mixed lineage leukemia (MLL) fusion signals were detected with positive rates of 96.5 and $6 \%$, respectively. (A) PML/RARA fusion signals are indicated by a yellow signal (PML in red and RARA in green). (B) Using an MLL break-apart rearrangement probe, a cell lacking the MLL rearrangement exhibits a two red/green (yellow) fusion signal pattern. In a cell possessing an MLL translocation, the expected pattern is one green/red (yellow) fusion signal, one red signal and one green signal.

blood test was markedly abnormal, with a white blood cell count of $66.92 \times 10^{9} / 1$ (normal range, 4.0-10.0× $10^{9} / 1$ ), $20 \%$ neutrophils (normal range, 50]70\%), a hemoglobin level of $102 \mathrm{~g} / 1$ (normal range, 110-150 g/l) and a platelet count of $61 \times 10^{9} / 1$ (normal range, 100-300x109/1). Physical examination revealed scattered ecchymosis on the limbs, bilateral cervical part lymphadenopathy and strengthened muscular tension of limbs.

Blood chemistry on admission showed elevated lactate dehydrogenase (1,142 U/l; normal range, 104-245 U/1), alanine (92 U/1; normal range, 10-40 U/1) and aspartate aminotransferase (96 U/1; normal range, 10-40 U/l) levels. The total protein, albumin, serum creatinine and blood urea nitrogen levels were within normal limits. The prothrombin time was $14 \mathrm{sec}$ (normal range, 11-13 sec), the activated partial thromboplastin time was $20.7 \mathrm{sec}$ (normal range, 31-43 sec), the thrombin time was $25.3 \mathrm{sec}$ (normal range, 16-18 sec), the fibrinogen level was reduced to $0.83 \mathrm{~g} / 1$ (normal range, 2.0-4.0 g/l), the protamine paracoagulation test was positive and D-dimers were increased to $2.894 \mathrm{mg} / 1$ (normal range, $0 \pm 0.3 \mathrm{mg} / \mathrm{l})$. BM aspiration revealed that $>76 \%$ of marrow cells were abnormal promyelocytic cells (Fig. 1). Translocations involving the mixed lineage leukemia (MLL) and promyelocytic leukemia/retinoic acid receptor- $\alpha$ (PML/RARA) genes were detected by fluorescence in situ hybridization (Fig. 2). An abdominal computed tomography scan showed slight splenomegaly. The final diagnosis was of post-traumatic brain syndrome and APL. The patient was treated with all-trans retinoic acid $\left(25 \mathrm{mg} / \mathrm{m}^{2} /\right.$ day $)$, and cryoprecipitate was transfused for correction of the DIC. However, the patient's condition deteriorated due to a severe infection of the lung and uncontrolled gastrointestinal bleeding caused by the DIC. Thus, the patient succumbed 10 days later.

\section{Discussion}

TBI always destroy neurons, glial cells, nerve fibers and blood vessels directly, and the effective treatment of neurological impairment has been a widespread problem in clinical practice (7). Early studies suggested that the CNS had no ability to renew or regenerate after injury. This viewpoint has gradually been corrected through the research progress in the fields of stem cells and neuroregeneration (9). The compensation from uninjured neurons, and the migration and differentiation of neural stem cells may contribute to the neurological recovery of patients with TBI. However, the capability of self-recovery is weak due to the limited quantity of a patient's own stem cells. Therefore, exogenous stem cell transplantation provides a novel method of promoting the recovery of neurological function in patients with TBI (10).

A number of studies found that MSCs can give rise to non-mesenchymal cell types, such as glial cells, neurons and hepatocytes, among others (1). This provides a promising method for replacement therapy. Transplanted MSCs may confer beneficial effects in patients with CNS injuries by potential mechanisms such as the migration to injured tissues, transdifferentiation to replace neural cells that are damaged and the induction of growth factor production (1). For example, neuronal MSC differentiation could provide a source of cells for the replacement of neurons that are lost due to neurodegenerative diseases. Studies have shown that neurons differentiated from MSCs exhibit functional 
neuronal properties. The major cell types used in clinical treatment are neural stem cells, BMMSCs and umbilical cord MSCs (9). Compared with the other cell types, autologous BMMSCs avoid ethical controversy and immune rejection, and can be easily obtained through repeated harvests (7). Hence, BMMSCs has become an important source of seed cells for the treatment of a wide variety of nervous diseases.

Although the major continued investigation of MSCs may assist in ensuring that cell based-therapy is used safely and effectively in human disease, there is growing concern over the clinical use of MSCs. Since MSCs are multipotent, one issue that requires addressing further is the potential tumorigenesis (11). Theoretically, MSCs are also known to home in on tumors, and once set up in the tumor microenvironment, they are able to support the growth of the tumor and spread. Recent studies have also demonstrated that newly injected MSCs often travel back to the BM, to inflamed tissues/organs or to sites of growing tumors, indicating that besides their functions in tissue repair, MSCs are important in immunity modulation and tumor growth (11). The inflammation-cancer link has been known for decades; MSC mobilization/activation in vivo in response to the damage or inflammation of tissues may enable the growth of pre-cancerous or dormant tumors (12). MSCs exhibit tumor-promoting functions by physically providing tumor-nurturing niches when recruited to the sites of growing tumors. Studies have also suggested that MSC-derived soluble molecules, including fibroblast-specific protein 1, stromal cell-derived factor- $1 \alpha$, chemokine (C-C motif) ligand 5, interleukin 6 and chemokine (C-X-C motif) ligand 7, are key to this tumor promotion, providing a mechanism by which tumor-promoting molecules are secreted by MSCs in a paracrine manner (12). In addition, MSCs have been shown to favor angiogenesis, which also promotes ovarian tumor growth. Biochemical analysis suggests that MSC-conditioned medium induces VEGF expression in tumor cells (12). Furthermore, another critical issue with regard to tumor promotion is the immunosuppressive prosperity of MSCs. MSCs are known to affect the proliferation and differentiation of dendritic cells, $\mathrm{B}$ and $\mathrm{T}$ cells, monocytes/macrophages, natural killer cells and mast cells (11).

Recipients receiving organ or cell transplantation are susceptible to leukemia, which may be attributed to donor-derived leukemic cells, the use of cytotoxic agents and long duration immunosuppression, but the exact mechanism has yet to be elucidated (13). Immunosuppression is believed to be the most significant risk factor for malignancy following transplantation. In the present case, APL occurred early after autologous BMMSC transplantation, which is extremely rare. Previous studies have reported that acute leukemia did not occur between 2 months and 17 years post-organ transplantation $(14,15)$. There are a number of hypotheses regarding the development of leukemia in recipients with autologous BMMSC transplantation. The majority of reported cases of leukemia following transplantation have been associated with a cytogenetic abnormality (16). Camós et al (15) demonstrated that the chromosomal alterations that are typically identified in therapy-related AML, such as monosomy or deletion of chromosomes 5 and 7 , and $11 \mathrm{q} 23$ rearrangements, were not found in these AML cases. However, APL with $\mathrm{t}(15,17)$ translocation is the most common subtype of AML, even though few AML cases have been reported following liver transplantation (15). AML after solid organ transplantation is rare and, to date, only 9 cases of this form of leukemia after liver transplantation have been described, including 3 cases of APL and 6 with normal karyotypes. The present patient was diagnosed with APL with PML/RARA translocation and MLL rearrangement, also supporting this viewpoint of leukemogenesis after cell therapy. Another possibility is immunosuppression, where the potentially leukemogenic factor may play a role in developing AML after transplantation. The impaired immunosurveillance caused by the immunosuppressive effect of MSCs may be responsible for an increased incidence of leukemia.

In conclusion, the present patient developed de novo APL following autologous BMMSC transplantation for TBI. Although the precise mechanism could not be identified, the cytogenetic abnormality and the immunosuppressive effect of the MSCs may contribute to this leukemogenesis. Despite this, MSC transplantation is a promising treatment for TBI, although the safety of MSC application remains a challenging issue that requires further investigation.

\section{References}

1. Zhang R, Liu Y, Yan K, Chen L, Chen XR, Li P, Chen FF and Jiang XD: Anti-inflammatory and immunomodulatory mechanisms of mesenchymal stem cell transplantation in experimental traumatic brain injury. J Neuroinflammation 10: 106, 2013.

2. Mauritz W, Wilbacher I, Majdan M, Leitgeb J, Janciak I, Brazinova A and Rusnak M: Epidemiology, treatment and outcome of patients after severe traumatic brain injury in European regions with different economic status. Eur J Public Health 18: 575-580, 2008.

3. Scudday T, Brasel K, Webb T, Codner P, Somberg L, Weigelt J, Herrmann D and Peppard W: Safety and efficacy of prophylactic anticoagulation in patients with traumatic brain injury. J Am Coll Surg 213: 148-153, 2011

4. Parsons XH, Teng YD, Parsons JF, Snyder EY, Smotrich DB and Moore DA: Efficient derivation of human neuronal progenitors and neurons from pluripotent human embryonic stem cells with small molecule induction. J Vis Exp 28: e3273, 2011.

5. Centeno CJ, Schultz JR, Cheever M, Freeman M, Faulkner S, Robinson B and Hanson R: Safety and complications reporting update on the re-implantation of culture-expanded mesenchymal stem cells using autologous platelet lysate technique. Curr Stem Cell Res Ther 6: 368-378, 2011.

6. Dai G, Liu X, Zhang Z, Yang Z, Dai Y and Xu R: Transplantation of autologous bone marrow mesenchymal stem cells in the treatment of complete and chronic cervical spinal cord injury. Brain Res 1533: 73-79, 2013.

7. Tian C, Wang X, Wang X, Wang L, Wang X, Wu S and Wan Z: Autologous bone marrow mesenchymal stem cell therapy in the subacute stage of traumatic brain injury by lumbar puncture. Exp Clin Transplant 11: 176-181, 2013.

8. Wakitani S, Okabe T, Horibe S, Mitsuoka T, Saito M, Koyama T, Nawata M, Tensho K, Kato H, Uematsu K, et al: Safety of autologous bone marrow-derived mesenchymal stem cell transplantation for cartilage repair in 41 patients with 45 joints followed for up to 11 years and 5 months. J Tissue Eng Regen Med 5: 146-150, 2011.

9. Meirelles Lda S and Nardi NB: Methodology, biology and clinical applications of mesenchymal stem cells. Front Biosci (Landmark Ed) 14: 4281-4298, 2009.

10. Wang S, Cheng H, Dai G, Wang X, Hua R, Liu X, Wang P, Chen G, Yue W and An Y. Umbilical cord mesenchymal stem cell transplantation significantly improves neurological function in patients with sequelae of traumatic brain injury. Brain Res 1532: 76-84. 2013

11. Waterman RS, Henkle SL and Betancourt AM: Mesenchymal stem cell 1 (MSC1)-based therapy attenuates tumor growth whereas MSC2-treatment promotes tumor growth and metastasis. PLoS One 7: e45590, 2012. 
12. Zhu W, Huang L, Li Y, Qian H, Shan X, Yan Y, Mao F, Wu X and Xu WR: Mesenchymal stem cell-secreted soluble signaling molecules potentiate tumor growth. Cell Cycle 10: 3198-3207, 2011.

13. Liu M, Liu J, Liu L, Yu L, Shi B, Ye L, Zhang Y and Chen H: A case report of acute myeloid leukemia after liver transplantation. Acta Haematol 129: 225-228, 2013.

14. Jiang N, Li H, Wang GS, Zhang J, Zhang JF, Yi SH, Yang Y, Cai CJ, Lu MQ and Chen GH: Acute leukemia, a rare but fatal complication after liver transplantation. Leuk Res 33: 1349-1351, 2009.
15. Camós M, Esteve J, Rimola A, Grande L, Rozman M, Colomer D, Villamor N, Costa D and Montserrat E: Increased incidence of acute myeloid leukemia after liver transplantation? Description of three new cases and review of the literature. Transplantation 77: 311-313, 2004.

16. Sato T, Kobayashi R, Iguchi A, Nakajima M, Koizumi S, Furukawa H, Todoh S and Kobayashi K: Acute promyelocytic leukemia after living donor partial orthotopic liver transplantation in two Japanese girls. Leuk Lymphoma 46: 1057-1060, 2005 . 